811.112.2’367.635

https://doi.org/10.18485/sj.2019.24.1.14

DANICA B. NEDELJKOVIĆ*

Universität Kragujevac

Fakultät für Philologie und Künste

Lehrstuhl für Germanistik
Оригинални научни рад

Примљен: 11. 09. 2018.

Прихваћен: 15. 01. 2019.

\title{
KOPULAPARTIKELN IN DER DEUTSCHEN GEGENWARTSSPRACHE ${ }^{* *}$
}

Das Ziel des vorliegenden Beitrags ist es, den Status von sprachlichen Einheiten, die im Dependenzmodell von Ulrich Engel aufgrund deren Unflektierbarkeit und deren auf den prädikativen Gebrauch beschränkter syntaktischer Funktion als Kopulapartikeln definiert werden, anhand von Beispielen aus Pressetexten zu analysieren und das Inventar dieser Wortart im Gegenwartsdeutsch zu bestimmen. Es werden vor allem Lexeme in den Blick genommen, die in der (nicht nur gesprochenen) Gegenwartssprache die Tendenz aufweisen, aus der Klasse der unflektierbaren in die der flektierbaren Wörter überzutreten und damit auch in unterschiedlichen syntaktischen Realisationen zu erscheinen, wodurch sie den ursprünglich angenommenen Status von Kopulapartikeln verlieren. Die durchgeführte Analyse bestätigt die Ausgangshypothese, dass sich das Inventar von Kopulapartikeln angesichts der beschriebenen Tendenz drastisch reduziert hat und dem Kenntnisstand in der einschlägigen Fachliteratur nicht mehr entspricht.

Schlüsselwörter: Kopulapartikel, Adjektiv, Adverb, Valenz, Deutsch.

*dbmarjan@eunet.rs

** Die vorliegende Arbeit wurde im Rahmen des Projekts178014: Dinamika struktura savremenog srpskog jezika angefertigt, das vom Ministerium für Bildung, Wissenschaft und technologische Entwicklung der Republik Serbien finanziert wurde. 


\section{EINLEITUNG}

1.1. Sprache ist kein unveränderliches System, sie wird täglich neu und kreativ verwendet, um allen unseren kommunikativen Bedürfnissen gerecht zu werden (Denkler et al. 2008: 7). Sprachliche Veränderungen beginnen meist als Abweichungen von der Norm, d.h. als Regelverletzungen, die man „Fehler” nennt. Wenn sich aber der fehlerhafte Gebrauch verbreitet und schließlich zum allgemeinen Usus wird, wird er nicht mehr als Fehler betrachtet, sondern als eine neue Konvention (Keller 2006: 196). Daraus folgert der Autor: „Die systematischen Fehler von heute sind die neuen Regeln von morgen.” (ebd. 199). In diesem Licht lässt sich auch die Problematik der Wortart Kopulapartikeln betrachten.

1.2. Den Gegenstand der vorliegenden Untersuchung bilden die sprachlichen Einheiten, die in der dependentiell ausgerichteten Fachliteratur aufgrund ihrer Unflektierbarkeit und ihrer auf das Prädikativum beschränkten syntaktischen Funktion als eine eigene Wortklasse - Kopulapartikeln - definiert werden. Da sich aber im gegenwärtigen (nicht nur gesprochenen) Deutsch die steigende Tendenz zur Abweichung von den oben genannten Kriterien bemerkbar macht, wird in diesem Beitrag von der Hypothese ausgegangen, dass viele der ursprünglich als Kopulapartikeln definierten Lexeme ihren Status inzwischen geändert haben und in eine andere Wortklasse übergetreten sind, wodurch sich das Inventar von Kopulapartikeln deutlich reduziert hat.

1.3. Das Ausgangskorpus für die Untersuchung bilden die deutschen Grammatiken, die die Kopulapartikeln als eine selbstständige Wortklasse unterscheiden: Kontrastive Grammatik Deutsch-Serbokroatisch (1986) von U. Engel und P. Mrazović, Deutsche Grammatik (1988) von U. Engel, Deutsche Grammatik - Neubearbeitung (2004) von U. Engel sowie Deutsch-serbische kontrastive Grammatik, Teil IV: Partikeln (2017) der Autorengruppe Đurović / Ivanović / Srdić / Engel / Alanović. Das Kontrollkorpus bilden die kontextualisierten Beispiele aus Pressetexten (teils DWDS-Zeitungskorpora, teils Zeitungsartikeln aus dem Internet entnommen), die die Zugehörigkeit der zu erforschenden sprachlichen Einheiten zu den Kopulapartikeln bestätigen oder in Frage stellen sollen. In einem ersten Schritt wird eine Liste von sprachlichen Einheiten erstellt, die in den oben genannten Grammatiken als Kopulapartikeln definiert werden. Die älteren und die neueren Auflagen gleicher Autoren bzw. Grammatiken ermöglichen es, die von den Autoren selbst durchgeführten Änderungen in den später erschienenen Auflagen zu verfolgen. In einem zweiten Analyseschritt werden solche sprachlichen Einheiten auf die syntaktische Funktion sowie Flektierbarkeit hin untersucht, um diejenigen, die der Definition von Kopulapartikeln nicht mehr entsprechen, aussondern zu können. Auf diese Weise sollen der aktuelle Status und das Inventar von Kopulapartikeln im Gegenwartsdeutsch präzise bestimmt werden. In einem dritten Schritt wird die Valenz der Kopulapartikeln thematisiert, da sie auch phrasenbildend sind und sowohl Ergänzungen als auch Angaben regieren können. 


\section{KOPULAPARTIKELN ALS WORTKLASSE}

2.1. In der Dependenzgrammatik von U. Engel gilt der Begriff Partikeln einerseits als Oberbegriff für alle unflektierbaren Wortklassen und andererseits bezeichnet er unterschiedliche Subklassen der Unflektierbaren (Engel 1988: 18f.). Eine davon sind die Kopulapartikeln, die sich von den anderen Wortarten, vor allem aber Adjektiven und Adverbien, dadurch unterscheiden, dass sie weder in attributiver noch in adverbialer Funktion auftreten können. Sie kommen also lediglich prädikativ vor: „Kopulapartikeln sind unveränderliche Wörter, die als Adjektivalergänzung [...] jedoch nicht als Attribut zum Nomen verwendet werden können" (Engel 1988: 767). Die Bezeichnung Kopulapartikeln wird erstmals von U. Engel eingeführt und dadurch erklärt, dass solche sprachlichen Einheiten von den Kopulaverben ${ }^{1}$ (sein, werden, bleiben, scheinen) und wenigen anderen (z.B. finden, gehen, machen, tun) regiert werden. Dabei wird betont, dass sich keine der Kopulapartikeln mit allen oben genannten Verben verbinden lässt, es ist vielmehr so, dass sich viele nur mit einem kombinieren lassen (ebd.). Zu den Kopulapartikeln werden u.a. folgende Lexeme gerechnet (vgl. Engel und Mrazović 1986: 921ff.; Engel 1988: 767; 2004: 421; Đurović et al. 2017: 97): abhold, abspenstig, angst, anheischig, ausfindig, egal, einerlei, feind, getrost, gewahr, gewillt, gram, handgemein, leid, los, perplex, pleite, quitt, schade, schuld, teilhaftig, untertan, vorstellig, zugetan u.a. Darüber hinaus werden als Kopulapartikeln auch einige stilistisch als umgangssprachlich (ugs.) oder alltäglich (allt.) markierte Lexeme aufgeführt, wie z.B.: futsch, k.o., meschugge, o.k. / okay / okey /O.K., pleite, plemplem, schnuppe u.a. sowie einige Wortpaare / Zwillingsformen (angst und bange, fix und fertig, gang und gäbe, klipp und klar, los und ledig, null und nichtig u.a.). Die letztgenannten mussten aufgrund des beschränkten Umfangs dieser Arbeit außer Acht gelassen werden.

2.2. In der einschlägigen Literatur sind neben dem Terminus Kopulapartikeln auch andere Termini geläufig. So wird in der IDS-Grammatik (Zifonun et al. 1997: 55) für diese Wortklasse die Bezeichnung Adkopula verwendet ${ }^{2}$. Demgegenüber ist man in der traditionell orientierten Literatur zum großen Teil der Auffassung, dass solche Elemente keine selbstständige Wortklasse bilden, sondern als „,nur prädikativ verwendbare Adjektive" zu betrachten sind (Duden 2005: 365f.; Wahrig 2009: 353f.; Hentschel und Weydt 2003: 209). Des Weiteren wird von den „monoprädikativen Zustandsadjektiven" als einer neuen Adjektivklasse, die sich durch ausschließlich

${ }^{1}$ Kopulaverb ist ein Terminus der traditionellen Grammatik und bezeichnet die Verben, die „nur eine sehr allgemeine Bedeutung haben und im wesentlichen als Verbindungsmittel (lat. copula) zwischen dem Subjekt und einer Adjektival- oder Nominalergänzung (traditionell: einem Prädikatsnomen) dienen" (Engel 1988: 767).

${ }_{2}^{2}$ „Die ADKOPULA (fit, gewillt, leid, pleite, quitt, schade, schuld, ...) bildet gemeinsam mit einem Kopulaverb den Ausdruck des minimalen Prädikats. Sie ist Komplement der Kopula und auf diese Funktion spezialisiert, kann also - anders als die auch in dieser Funktion vorkommenden Adjektive - nicht attributiv verwendet werden und ist nicht flektierbar." (Zifonun et al. 1997: 55) 
prädikative Verwendung auszeichnet, gesprochen (Trost 2006: 151ff.) ${ }^{3}$. Beide Bezeichnungen widersprechen der Adjektivdefinition von U. Engel (1988: 556), nach der Adjektive Wörter sind, „die zwischen Determinativ und Nomen stehen können", also die Einsetzprobe Det Nom bestehen können.

Von der vorangehenden Definition ausgehend scheint die Differenzierung der Klasse Kopulapartikeln völlig berechtigt zu sein. Problematisch wird in diesem Kontext bloß das Inventar dieser Wortart, denn es wird des Öfteren angemerkt, dass viele der als Kopulapartikeln definierten Lexeme auch andere syntaktische Funktionen im Satz, vor allem attributive, ohne Bedeutungsunterschied ausüben können, z.B. abspenstig, abhold, getrost, gewillt, untertan u.a. (Römer 2006: 162; Trost 2006: 151f.; Castell 2016: 180ff.). In der Duden-Grammatik (2005: 365f.) wird hervorgehoben, dass einige dieser ,nur prädikativ gebrauchten Adjektive” (z.B. fit, kaputt, tipptopp) vor allem umgangssprachlich auch attributiv gebraucht und dann auch flektiert werden können (ein fitter Bursche, das kaputte Fahrrad, eine tipptoppe Arbeit) oder dass ihr attributiver Gebrauch auf die dichterische Freiheit zurückzuführen ist (in gang und gäber Schwärmerei, Th. Mann), während manche auch im adverbialen Gebrauch erscheinen können (Sie spielten barfuß.; Die Elf hat klasse gespielt.). Zum gleichen Schluss kommen selbst die Befürworter der Klasse Kopulapartikeln (Engel 1988: 768, Zifonun 1997: 55; Đurović et al. 2017: 97, Hrustić 2006: 130-146), die aber aufgrund der dominierenden prädikativen Funktion und dem beschränkten Gebrauch in anderen syntaktischen Funktionen solche Elemente weiterhin zu den Kopulapartikeln rechnen - bis auf wenige Ausnahmen, die in den späteren Auflagen gleicher Autoren nicht mehr als Kopulapartikeln erscheinen und anderen Wortarten zugerechnet werden, z.B. barfuß, allein, irre, gar, fit (man vergleiche Engel 1986 und 1988 mit Engel 2004).

Ob die prädikative Funktion aus Sicht der Gegenwartssprache bei den als Kopulapartikeln definierten Lexemen weiterhin die einzige bzw. die dominanteste ist, wird in diesem Beitrag geprüft.

\section{SPRACHLICHE EINHEITEN, DIE DIE MERKMALE VON ADJEKTIVEN AUFWEISEN}

3.1. Die am Korpus durchgeführte Analyse hat gezeigt, dass sich aus der Liste der im Ausgangskorpus als Kopulapartikeln bezeichneten Lexeme folgende streichen lassen: abhold (4) ${ }^{4}$, abspenstig (4), anheischig (4), fit (3), getrost (3), gewillt (4), gram (4), perplex (4); teilhaft(ig) (3); untertan (4), vorstellig (4) und

${ }^{3}$ In Analogie dazu werden Adjektive, die nur attributiv verwendet weden können (z.B. hiesig, damalig) als „monoattributive Adjektive” definiert (Trost 2006: 1).

${ }^{4}$ Die in Klammern angegebene Zahl sagt darüber aus, wie oft das jeweilige Lexem im Ausgangskorpus als Kopulapartikel erscheint. Die Wörter, die in mindestens zwei der vier Grammatiken 
zugetan (4), da sie systematisch nicht nur prädikativ, sondern auch attributiv (ggf. auch adverbial) verwendet werden können. Zugegebenermaßen ist bei ihnen die prädikative Funktion immer noch die dominanteste (z.B. jemandem, einer Sache abhold sein; [irgendwo/bei jemandem/etwas] vorstellig werden) oder sie tauchen typischerweise als Bestandteil einer festen Wendung auf (z.B.j-m etw./j-n abspenstig machen, sich anheischig machen $)^{5}$, aber man darf die Tatsache nicht leugnen, dass auch der attributive Gebrauch durch Belege aus der Pressesprache nachgewiesen wurde. Vgl.:

(1) George W. Bush, 55, dem Alkohol inzwischen abholder US-Präsident, muss um sein ihm neu zugewachsenes Image als entschlossener Kriegsherr bangen. (www.spiegel.de; 18.02.2002); ... : die breite Zustimmung „ordentlicher”, angeblich jedem Extrem abholder Bürger zu einem offen rassistischen Programm; ... (www.taz.de; 02.11.2004); Ganz vorn dabei sind natürlich die Berufsprovokateure von der AfD, allen voran der keiner Geschmacklosigkeit abholde Marcus Pretzell mit seinem „Merkels Tote!”-Tweet. (www.spiegel.de; 25.12.2016); Die Federführung übernahmen Bundesanwalt Herbert Diemer, ein zurückhaltender, jeder modischen Attitüde abholder Franke, und die Oberstaatsanwälte Anett Greger und Jochen Weingarten. (www.spiegel.de; 15.4.2013)

(2) So könnte es sein, dass die Grünen mit der Vereinnahmung des Freiheitsbegriffs gar nicht auf abspenstige FDP-Wähler zielen. (www.tagesspiegel.de; 09.01.2014); Sie hat sich ein Arsenal an Zwangsmaßnahmen gegen die abspenstigen Katalanen zurechtgelegt und droht nun damit, sich auf Artikel 155 der spanischen Verfassung zu berufen, ... (www.zeit.de; 11.10.2017); Das Integrationsgesetz der großen Koalition soll abspenstige Wähler zurückholen und populistische Strömungen in der Union befrieden. (www.welt.de; 14.04.2016); Auch ihr Mitorganisator René Jahn, ..., appelliert an die abspenstigen Demonstranten. (www.spiegel.de; 06.01.2015)

(3) Die anheischigen „Toleranz-, Wertschätzungs- und Respekt-Kulturen” dienen als Einfallstore für beliebige Forderungen. (http://frankfurter-erklaerung.de; 3.4.2018); Gysi antwortet mit anheischigem Ton: „Aber ich habe meinen Job doch gut gemacht, oder?” (www.focus.de; 9.8.2015); „Nein, mein lieber Volkskamerad Flutsch” (Frauke versucht ihren Ekel vor dem immens anheischigen Schrumpfgermanen zu verbergen), .... (www.wuerzburcher.de; 5.10.2016); Der anheischige Kunstbetrieb wird immer mehr zu einer Ausrede für das, was fehlt: Aufklärung. Und zwar nicht durch die Polizei. (https://derstandard.at, 2.2.2005)

(4) Es ist ein Schock, wenn junge, fitte Menschen wie "Kiss FM"-Moderator Sebastian Radke plötzlich tot umfallen. (www.focus.de; 10.04.2015); 10 Lebensmittel, die fitte Menschen lieben. Wir alle würden gerne die Geheimnisse fitter Leute kennen. (www.gesundheute. com); Gestresste Mama, fittes Kind (www.focus.de; 17.5.2006); „Die Schere zwischen sehr fitten Kindern und solchen, die sich überhaupt nicht bewegen, öffnet sich immer weiter", ... (www.spiegel.de; 21.3.2017) ${ }^{6}$

(5) Ein Tag des getrosten Ausblicks auf eine gesegnete Zukunft (www.kreiszeitung. de; 16.6.2015); Und alle Zweifler, die sich fragen, ob Schauspiel und Performance zu vereinen wären, dürfen getrosten Mutes sein: Das Aus- und Vorstellen verträgt sich bestens.

(aus den Jahren 1986, 1988, 2004 und 2017) als solche definiert werden, werden in das Korpus aufgenommen.

${ }^{5}$ Für das attributive und das absolute Vorkommen jedes einzelnen Wortes sowie die Häufigkeit dessen Gebrauchs vgl. Castell 2016: 181f.

${ }^{6}$ In attributivem Gebrauch sowie bei Steigerungsformen kommt es zur Verdoppelung des Konsonanten $t$ (vgl. Duden: ein fitter Bursche; die fitteste Läuferin wird gewinnen; Steigerungsformen: fitter, fitteste). 
(www.fr.de; 17.11.2017); Vielmehr soll die Feldseelsorge (...) durch möglichst enge Fühlung mit allen Teilen der Truppe und ihrer Führung um die seelische Haltung der Soldaten und um ihre Ausrüstung mit tapferem und getrostem Glauben bemüht sein. (www.linksnet.de; 26.9.2008); Nach einem erfüllten Leben voller Hingabe und Liebe entschlief heute im getrosten Glauben an ihren Herrn und Erlöser im 92. Lebensjahr unsere liebe Mutter, ... (https://trauer.zevenerzeitung.de; 4.3.2016)

(6) Dazu aber brauchte sie die Zustimmung des Kronrats und die eines gewillten Parlaments. (Die Zeit, 06.02.2014, Nr. 07); Vor allem traten die Wolfsburger einen Tag nach dem Offenen Brief an ihre Fans (...) endlich auch wieder wie ein geschlossenes und sichtlich gewilltes Team auf. (Die Zeit, 18.02.2011, Nr. 8); Sollten andere Länder den Druck auf den Irak nicht aufrechterhalten und Bagdad selbst sich weiterhin der Welt widersetzen, würden die USA eine „Koalition anderer gewillter Nationen anführen”, um Irak zu entwaffnen, sagte Bush. (www.spiegel.de, 29.1.2003); „Einigung Europas ... unter Einbeziehung Großbritanniens und anderer zum Beitritt gewillter Staaten". (Die Zeit, 16.01.2003, Nr. 04) ${ }^{8}$

(7) Im tiefsten Inneren sind sie kinderlos, grame Verkörperungen der deutschen Weltskepsis, Zukunftsangst und Unfruchtbarkeit. (Die Zeit, 03.06.2004, Nr. 24); Während sich die Meinungen darüber gerade verfestigen, so könnte man ihn interpretieren, rutschen die beiden einander gramen Tanten im Schlaf immer näher aufeinander zu. (Die Zeit, 20.03.2003, Nr. 13); Hier draußen vor den Toren der Stadt, in einem gepflegten Park hinter hohen Backsteinmauern, hatte sich der grame Oberst Perón verkrochen, um nach dem Tode seiner so sehr vom Volk geliebten Evita alleine weiterzuregieren. (Die Zeit, 03.05.1991, Nr. 19); Zu zahlreich sind wir Lebenden geworden, als dass noch Raum wäre für das ,uns grame Geschlecht jener Gespenster, die demjenigen begegnen, der schwerer als andere an der dem Menschen aufgebürdeten Trauerlast zu tragen hat. (Der Tagesspiegel, 02.05.2004)

(8) Perplexe Jazzfreunde versuchen die Takte mitzuzählen, lassen es aber bald, weil in der hochtourigen Mixtur alles Erdenkliche an Rhythmen enthalten ist. (Die Zeit, 12.11.2015, Nr. 46); «Was ist denn da passiert?», fragte die perplexe Mercedes-Rennleitung. (Die Zeit, 23.09.2012 (online)); Deshalb die perplexe Reaktion der beiden Regierungen, als der Vorschlag vor Ostern auf ihre Schreibtische flatterte. (Der Tagesspiegel, 29.04.2003); Fünf Tore in neun Minuten", erklärte der perplexe Bayern-Coach, der nach dem fünften Treffer seines TORnados fassungslos die Hände über dem Kopf zusammenschlug. (Die Zeit, 23.09.2015 (online))

(9) Schließlich ist auch der Hintergrund des industriell organisierten NS-Massenmords an angeblich wertlosen, menschlicher Würde nicht teilhaftigen Menschen zu sehen. (Die Zeit, 30.08.2017 (online)); Und meldete sich solch ein der Gnade des Vergessens nicht mehr

${ }^{7}$ Attributives getrost taucht vorwiegend in Religionstexten oder als Bestandteil der Wendung getrosten Mutes auf, welche als periphrastische Form des Adjektivs getrost zu betrachten sei. Das Adjektiv getrost ist von der homonymen Abtönungspartikel getrost zu unterscheiden, das als Synonym zu ruhig gebraucht werden kann (Castell 2016: 184), z.B.: Man kann dies getrost als Sabotage der Klimaforschung verstehen. (www.zeit.de; 26.3.2017); Doch das Foltergefängnis Tadmur bei Palmyra in der Wüste kann man getrost als Konzentrationslager bezeichnen. (Die Zeit, 04.03.2017, Nr. 08); Dass demnächst eine Milliarde Smartphones und Tablets akut gefährdet sind, darf man also getrost als Übertreibung von Zimperium ansehen. (www.zeit.de; 1.10.2015).

${ }^{8} \mathrm{Zu}$ bemerken ist die Inkonsequenz in der Darstellung des Lexems gewillt bei U. Engel, der es einerseits als Kopulapartikel bezeichnet (1988: 767; 2004: 422), andererseits aber zu den Adjektiven mit Verbativergänzung rechnet (1988: 594), wobei er u.a. folgendes Beispiel aufführt: ?mein nicht mitzuarbeiten gewillter Bruder. Das vorangestellte Fragezeichen soll von der fraglichen Grammatikalität dieses Gebrauchs zeugen, ist aber in der Neubearbeitung seiner Grammatik (2004: 355) nicht mehr vorzufinden, nämlich in dem Beispiel eine da zu bleiben gewillte Genossin (dazu vgl. auch Castell 2016: 180). Darüber hinaus sei an dieser Stelle vermerkt, dass im letzten unserem Korpus entnommenen Beispiel gewillt keine Verbativ-, sondern eine Präpositivergänzung regiert (anderer zum Beitritt gewillter Staaten). 
teilhaftiger, volldigitaler Savant, so müßte man ihn an ein Unterhaltungsquiz des ZDF weiterempfehlen, ... (Die Zeit, 08.01.1988, Nr. 02); Einmal an einem besonderen Ort gemeinsam essen und beten und dabei das Licht auf das Selbstverständnis unserer teilhaftigen priesterlichen Berufung fallen lassen. (www.meinbezirk. at; 26.2.2013); Er sei aber davon überzeugt, ,,dass es ständig und ohne Unterlass eines der menschlichen Absurdität teilhaftigen Sterblichen" bedürfe, der die richtigen Fragen stelle. (www.derstandard.de; 12.11.2017)

(10) Denn es ist genau jenes bauwütige, dem blinden Wachstum untertane China, vor dem im Westen immer gewarnt wird. (Die Zeit, 12.07.2001, Nr. 29); Er präsentierte sich als Staatsmacht - das musste greifen, da das allzeit untertane Ostvolk zwar nicht seine gestrigen Bedrücker vermisste, jedoch die regelstiftende Obrigkeit. (Die Zeit, 27.01.2000, Nr. 5); Diese Religion würde durch Kaiser Friedrich wieder hergestellt werden, und ein ihm untertaner Patriatch in Mainz den Papst ersetzen. (www.zauberspiegel-online.de); Für den niemand untertanen Autonomen, der sich selbst bestimmt. (Die Zeit, 18.02.1999, Nr. 08)

(11) Sollten nicht beide Erziehungsberechtigten zur Anmeldung kommen können, muss der vorstellige Erziehungsberechtigte eine Vollmacht des verhinderten Elternteils mitbringen. (www.ruhrnachrichten.de; 29.1.2015); Damit können Arztpraxen sicherstellen, dass der vorstellige Patient auch tatsächlich der Versicherte ist. (www.n-tv.de; 19.6.2012); Ort, Datum und Unterschrift der vorstelligen Person (www.wibank.de; Formular); Der Lebenswandel des durchaus zur Eitelkeit neigenen, alle drei Wochen beim Friseur vorstelligen Jungstars, ..., wurde zudem als unstet beschrieben. (www.fr.de; 24.11.2008) ${ }^{9}$

(12) Sogar der dem Rampenlicht ansonsten eher zugetane Klopp, ..., empfindet den Trubel als übertrieben: (Die Zeit, 06.04.2016 (online)); Eine jüngere Kollegin aus einem der Wirtschaft nicht besonders zugetanen Ressort dieser Zeitung bemerkte vor Kurzem, das Wort "Altersvorsorge« mache ihr schlechte Laune, mehr noch, es bereite ihr Angst. (Die Zeit, 15.11.2012, Nr. 47); Aber selbst dem Präsidenten zugetane US-Medien reagierten zurückhaltend auf die mit Spannung erwartete Ansprache. (Die Zeit, 16.06.2010 (online)); 1907 als Henriette Markovitch geboren, fand die den Surrealisten zugetane Fotografin im Paris der zwanziger Jahre Motive und Anregungen in Hülle und Fülle. (Die Zeit, 29.11.2001, Nr. 49)

Im Gegensatz zu den oben aufgelisteten Lexemen konnten die im Ausgangskorpus auch als Kopulapartikeln klassifizierten Lexeme gewahr (3) und handgemein (3) im attributiven Gebrauch deutlich seltener bzw. nur sporadisch, oft auch nur textsortenspezifisch belegt werden. Dies lässt sich mit der Tatsache erklären, dass sie stilistisch markiert sind und als veraltet und/oder gehoben gelten, daher aus gegenwartssprachlicher Sicht fast ausschließlich in festen Wendungen erscheinen. So ist beispielsweise gewahr als Bestandteil der Wendung jemanden, etwas / jemandes, einer Sache gewahr werden im Kontrollkorpus des Öfteren belegt worden, während der deutlich seltener vorkommende attributive (und adverbiale) Gebrauch fast ausschließlich auf die Texte religiösen und philosophischen Inhalts beschränkt ist (s. 13). Anders verhält es sich mit dem Lexem handgemein, das sogar in der Wendung [mit jemandem] handgemein werden in der Pressesprache kaum in Gebrauch ist (insgesamt gibt es nur 10 Belege für die genannte Wendung in den DWDS-Zeitungskorpora, Stand: 10.7.2018). Attributiv ist handgemein vor

${ }^{9}$ Da vorstellig für Papierdeutsch charakteristisch ist (Duden), ist es in der Pressesprache außerhalb der Wendung irgendwo / bei j-m vorstellig werden kaum vertreten, taucht aber in administrativen Texten (Formularen, Vorschriften u.Ä.) sowie in medizinischen Texten des Öfteren attributiv auf. 
allem in älteren Texten ${ }^{10}$ vorzufinden, in der gegenwärtigen Pressesprache dagegen überhaupt nicht. Ungeachtet dessen werden die beiden Lexeme, sowohl gewahr als auch handgemein, in diesem Beitrag zu den Adjektiven gerechnet, da sie die Möglichkeit des attributiven Gebrauchs nicht ausschließen und etymologisch als Adjektive zu interpretieren sind (s. DWDS), dazu noch gesteigert werden können (s. 20). Zur Illustration vergleiche:

(13) Gewalt rechtfertigende Steinbruchexegese wird getrennt von verantwortlicher, kritischer und des jeweiligen historischen wie theologischen Kontextes gewahrer Interpretation. (www.katholisch.de; 24.05.2016); Dieser Zustand - der eigentlich ein gewahrer Nicht-Zustand ist - erscheint dem denkenden Verstand nicht nachvollziehbar und wird möglicherweise auch Gefühle des Unbehagens auslösen. (www.konzert-der-stille.de; 16.4.2012); Mit gewahrer Achtsamkeit öffnen wir uns für die Felder, die unserer Seele begegnen möchten. (www.naturwissen.ch); Er war ein sich der eigenen Fehler allzu gewahrer, überaus selbstkritischer Idealist, das Gegenteil eines Pragmatikers. (fm4v2.orf.at/rotifer/220717/main.html; 13.11.2007)

(14) Die moralischen Statements in Ehren, aber es kann nicht sein das hier offen zu handgemeinen Aktionen geraten wird. (www.apfeltalk.de); Unter Gustav Adolf begann die Kavallerie sich wieder vornehmlich dem handgemeinen Einbruch in feindliche Formationen zu widmen, aber auch hier wurde vorher von den Pistolen Gebrauch gemacht, ... (https://m.hausarbeiten.de/document/146440); Auch diesmal waren die handgemeinen Angriffe aus dem hohlen Bauch heraus schiere Schikane der Gemeindeverwaltung gewesen- ... (www.plaetzchenwolf.eu/Vivarium48.html)

Von den für die Alltagssprache charakteristischen Lexemen, die im Ausgangskorpus als Kopulapartikeln bezeichnet werden, sind in attributiver Funktion folgende vorzufinden: meschugge (4), pleite (4), plemplem (3), futsch (4) und o.k. I okay / okey/O.K. (4). Während meschugge auch in der Pressesprache auftaucht (s. 15), konnten die anderen aufgrund der starken Gebundenheit an die Umgangssprache und der stilistischen Markiertheit als salopp oder ugs. (vgl. Duden, DWDS) nur in literarischen oder Pressetexten, die gesprochene Sprache wiedergeben sollen, belegt werden (z.B. o.k., plemplem) oder in Forums- und Blogbeiträgen, die bekanntlich durch ein hohes Maß umgangssprachlicher Ausdrücke gekennzeichnet sind (z.B. pleite, futsch). Auch wenn der attributive Gebrauch mancher dieser Lexeme von einigen Lexikographen als standardwidrig empfunden wird (man vergleiche z.B. Wahrig $2009^{11}$ ), ist die steigende Tendenz zum attributiven Gebrauch der genannten Wörter wenigstens in der informellen Kommunikation nicht zu leugnen und bedarf weiterer empirischer Untersuchungen. Es sei an dieser Stelle an das Adjektiv kaputt erinnert, dessen attributiver Gebrauch inzwischen als standardsprachlich gilt: das kaputte Radio, ein kaputter Auspuff (Wahrig 2009: 353f.). Zur Illustration vgl. folgende Belege:

${ }^{10}$ Man denke z.B. an das Hildebrandslied: „In handgemeiner Schlacht entscheide die Begegnung, Wer von uns heute die Harnische räumen müsse, ...”

${ }^{11}$ „Dieselbe Beschränkung gilt auch für einige umgangssprachliche Wörter: futsch, k.o., meschugge, o.k., plemplem, schnuppe. Für alle genannten Wörter gilt, dass ein attributiver Gebrauch nicht korrekt ist: *das futsche Geld, *ein meschugger Typ, *eine okaye Sache, *ein plemplemer Mensch, *eine schnuppe Meinung" (Wahrig 2009: 353f.). 
(15) Und Hans-Georg antwortet, dieser „Fiesel” sei ein „,meschugger Nilli” (will heißen: „der Mann dort ist ein verrückter Idiot”), wenn er wegen seines Stotterns mal wieder schräg angeguckt wird. (Die Zeit, 15.11.2001, Nr. 47); Die Dokumentation über den meschuggen Selbstfindungstrip, der - ... - den Wetteraurer Regisseur bis in den Himalaya führt, läuft zuletzt, am Sonntag um 21 Uhr. (www.welt.de; 18.3.2010); Es gab auch den „meschuggen SS-Mann”, der sich in die attraktive Frau Degen verliebt hatte und der noch kurz vor dessen Tod die Entlassung des Vaters aus dem KZ erwirkte. (Der Tagesspiegel, 16.02.2000); Ich habe es angenommen, weil ich schon so viele meschugge Sachen gemacht habe. (Berliner Zeitung, 09.10.1995)

(16) Ein völlig pleites Land mit 46 Millionen Einwohnern (die DDR hatte 17 Millionen und war lange nicht so pleite und am Boden wie die Ukraine) strebt in die EU. (www.spiegel. de/forum/; 26.10.2014); Was macht man mit einem pleitem (sic!) Betrieb? Warum verfährt man mit pleiten Ländern nicht wie mit pleiten Betrieben? Einfach das Personal entlassen und verkaufen. (www.forum-thueringen.de; 30.10.2012); Wie gut kann ein (beihnahe) pleites Unternehmen sein? (https://derstandard.at/; 16.4.2010); Man muss dazu wissen: RW Oberhausen gibt es nur deswegen noch, weil die pleiteste Stadt der Republik ihren Verein mit dem Kauf einer - gelinde formuliert überbewerteten - Immobilie rettete. (https://extradienst.net; 13.4.2017) ${ }^{12}$

(17) Dass die US-Amis ein komisches Völkchen sind, das nicht nur total plempleme sogenannte Präsidenten wählt, ..., sondern auch sonst recht durchgeknallt ist, das wurde jetzt auch am Beispiel des US-Staats Illinois ersichtlich. (www.journal.lu; 14.2.2017); Die Casting-Szene hatte ihren Hohepunkt im Auftritt von Heide Maier, die als vollkommen plempleme „Frau mit dem Stuhl" das Casting sprengte. (http://nicolefelden.de/inszenierungen/bei-ihnen-zuhause); Künstlerin bläst und wirbelt einem jederzeit allerlei anregende, paradoxe und wunderhübsch plempleme bis peinliche Ideen durch den Kopf. (www.taz.de; 18.2.2013); Ich mag plempleme Leute. Ich bin es selbst auch, auf andere Weise. (https://beziehung.gofeminin.de; 4.8.2010)

(18) Überlebt Marcel Koller die futsche WM-Quali? (www.newsjs.com; 3.9.2017); Die „Schwarz-Gelbe” Koalition ist abgewählt; und die „Futsche Partei Deutschlands” (FPD) hat endlich die verdiente Quittung für ihren Wirtschafts-, Markt- und Bankenlobbyismus erhalten. (www.soziales-netzwerk-deutschland-bad-kreuznach.de; 17.4.2014); Ich erinnere mich immer wieder gerne an den Spruch von Prof. Leo Brunnberg bei einer Podiumsdiskussion über die verschiedenen Operationstechniken zur Behandlung des vorderen Kreuzbandrisses beim Hund: „Ein futsches Knie bleibt futsch, unabhängig der Operationstechnik”. (https://procane-claudo. de/Rezension); Kommt ein Wanderer des Weges und singt den Anlegern, die ihr futsches Geld vermissen: ... (www.faz.net/; 2.10.2014)

(19) Kaltwasser ist neben Wagenbach der Einzige, der einen okayen Unterricht macht, aber während Wagenbach ein Arschloch ist, also menschlich, wird man aus Kaltwasser nicht schlau. (Tschick 53); Eigentlich eine ganz okaye Lüge, aber schon während ich sie dem Autobahnpolizisten zum ersten Mal auftischte, fiel mir ein, dass sie auffliegen würde. (Tschick 234); Charleen ist 15, hat eine ganz okaye Familie, eine beste Freundin - und einen Suizidversuch hinter sich. (www.spiegel.de; 6.8.2015); Und gibt es, wenn man das so vorsichtig sagen kann, eine okaye Art von Elternstolz? (www.zeit.de; 30.6.2018) ${ }^{13}$

${ }^{12}$ In der Wirtschaftssprache sind auch die Wendungen pleite gehen und pleite machen geläufig, vgl.: die Firma, das Handelshaus, Geschäft ist pleite (gegangen); das Theater hatte pleite gemacht (DWDS: pleite).

${ }^{13}$ In der Umgangssprache sind alle Formen vorzufinden: o.k. / okay / okey / O.K. In attributivem Gebrauch tauchen die gekürzten Formen entweder unflektiert (z.B.: Feines sushi für einen $o$. $k$. Preis im alten Palast in Split. (www.tripadvisor.de; 2.8.2017)) oder als erste Komponente eines Determinativkompositums auf (z.B.: Gute Lage und eine OK-Unterkunft (www.holidaycheck.de)). Flektiert kommen die Kurzformen mit einem Apostroph vor und sind stark umgangssprachlich (z.B.: Weniger Perfektionismus: Warum es mir hilft, eine $o k^{\prime} e$ Mutter zu sein (www.2kindchaos.com/mama/wenigerperfektionismus; 24.8.2017); Anders gesagt, ein ok'es Buch einer sehr erfolgreichen Rockband, ... (https://trust-zine.de/green-day-die-triografie-ben-myers/; 12.8.2008). 
3.2. Aus der durchgeführten Analyse lassen sich die Gründe gegen die Zuordnung aller im Absatz 3.1. behandelten Lexeme zu den Kopulapartikeln und für deren Zuordnung zu den Adjektiven ableiten. Sie sind morphologischer, syntaktischer und derivatologischer Natur.

Von den morphologischen Gründen ist zum einen zu erwähnen, dass einige dieser Lexeme die Form des Partizips II haben und etymologisch als solche zu interpretieren sind, z.B.: getrost, gewillt, untertan und zugetan (vgl. Duden). Partizipien lassen sich bekanntlich attributiv verwenden und verhalten sich in dieser Funktion wie Adjektive (vgl. dazu Petronijević 2011: 23f.). Des Weiteren ist aus der durchgeführten Analyse einsichtig, dass viele der als Kopulapartikeln bezeichneten Lexeme gesteigert werden können und in der Komparativ- und/oder Superlativform vorzufinden sind. Komparation ist eine Flexionsart, die auf die steigerbaren Adjektive beschränkt ist und als deren inhärentes Merkmal zu betrachten sei. Von den Unflektierbaren sind nur bei wenigen Adverbien Spuren einer Flektierbarkeit vorzufinden, die sich darin widerspiegelt, dass solche Adverbien kompariert werden können, z.B. oft - öfter/häufiger - am häufigsten; gern - lieber - am liebsten; bald-eher/früher-am ehesten/frühesten u.a. Wie aus den Beispielen ersichtlich, geht dies daraus hervor, dass „bei den Komparationsformen teils konkurrierend, teils ersatzweise Adjektivformen hinzutreten" (Engel 1988: 755). Demgegenüber gehören Kopulapartikeln zu den Unflektierbaren, die die Möglichkeit jeder Flexionsart ausschließen, was auf getrost, gewahr, gewillt, handgemein, meschugge, perplex, plemplem und pleite nicht zutrifft. Sie sind nämlich komparierbar, bilden die Steigerungsformen mit dem gleichen Stamm und können weder mit gleichbedeutenden adjektivischen Stämmen als Suppletivformen konkurrieren noch mit ihnen ersetzt werden. Aus allen oben genannten Gründen sind sie als Adjektive zu betrachten. Vgl.:

(20) „Länger und getroster leben” (www.deutschlandfunk.de; 18.6.2008.); Er legte ihnen nahe, das Leben und damit sich selbst getroster zu lieben. (Die Zeit, 16.10.1992, Nr. 43); Oder Spender, die ihr Geld für liberale Waffen-Gesetze investiert sehen möchten, überwiesen ihr Geld ebenfalls getroster an John McCain denn an Barack Obama. (Die Zeit, 04.11.2008, Nr. 45); Um die Vorweihnachtszeit noch besinnlicher zu begehen und sich der christlichen Traditionen wieder gewahrer zu werden, hat die Kirche die Adventszeit verdoppelt. (www.eine-zeitung.net; 28.10.2016); Im Oszillieren der Nacht wird man sich seiner eigenen Ausgesetztheit und Verletzbarkeit gewahrer, die auf die Grenzen des Handhabbaren im Dunklen verweisen. (www.feinschwarz.net; 7.4.2016); Womöglich ist man dort gewillter, die anti-israelische Stimmung weiter anzuheizen. (Der Tagesspiegel, 25.03.2001); Und entschieden handgemeiner geht es auf „Sonic Boom” denn auch zur Sache. (www.taz.de; 30.11.2009); Man hätte sich das gesamte Drehbuch etwas unberechenbarer, ja: „meschuggener gewünscht. (Der Tagesspiegel, 11.03.2004); Umso perplexer ist man, als der einsam wirkende Tischnachbar (Typ: zu große Jeansjacke) eine kurze Bemerkung zum Hofbräu, ..., zu einem abendfüllenden Vortrag über die Grundlagen des Brauens nutzt. (Die Zeit, 03.05.2012, Nr. 19); »Und du glaubst nicht, dass ich plemplem bin? «»Wer behauptet denn so etwas? «, fragte Blume. »Die anderen Kinder. « .... »Dabei sind die noch plemplemer.«Die Miene seiner Schwester verfinsterte sich. »Und wer ist am ... plemplemsten?« (www.weiterlesen.de/Leseprobe/Zeitlauscher $/ 9783942672429 / \mathrm{html}$ ); 
Wer laut schreit wird nur noch heiser, weil die Stadt endlich pleite ist. Berlin ist noch pleiter als die meisten anderen Länder. (www.tagesspiegel.de; 7.4.2001); Trotzdem waren die Länder noch pleiter als der Bund, die Kommunen am pleitesten. (Michel schlägt zurück: Satire By Dr.Jörg Hellmann)

Von den syntaktischen Gründen sei vor allem auf die Möglichkeit des adverbialen Gebrauchs hingewiesen, der neben dem attributiven und dem prädikativen die Wortart Adjektiv kennzeichnet ${ }^{14}$. Demgegenüber tauchen Kopulapartikeln laut Definition von U. Engel ausschließlich prädikativ auf, lassen also die anderen syntaktischen Funktionen nicht zu, was ein weiterer Grund dafür ist, die Lexeme getrost, gewahr, handgemein, perplex und pleite als Adjektive zu betrachten. Zur Illustration vgl.:

(21) So können wir wieder getrost einschlafen und im Traum mit Wolfi einen Achttausender erklimmen. (Die Zeit, 23.07.2003, Nr. 30); «Heute schon gelebt» - um getrost sterben zu können? (www.tagblatt.ch; 14.11.2015); Wahrscheinlich geisterte er deshalb durchs dunkle Saaletal: um den ersten Vorschein des Morgens zu erhaschen und dann getrost schlafen zu können. (Die Zeit, 08.03.2007, Nr. 11); „Länger und getroster leben” (www.deutschlandfunk.de; 18.6.2008.); Leitmotiv 2017: Gewahrer wahrnehmen was wirklich ist. Wahrnehmen und Denken ausserhalb vorgegebener Rahmen. (www.dasgelbeforum.net; 1.1.2017); Hast du den Wunsch gewahrer, bewusster und ganz präsent mit den Wesenheiten und Energien von der anderen Seite zu kommunizieren? (erichmatter.com/2018/06/11/ttte-2/); Und entschieden handgemeiner geht es auf ,Sonic Boom” denn auch zur Sache. (www.taz.de; 30.11.2009);"Weil ich schon gegessen habe", brachte ich perplex hervor. (Die Zeit, 05.11.2017 (online)); Im Fremdenverkehrsamt Jork schauten sie perplex, als ich vorhin danach fragte. (Die Zeit, 21.10.2017, Nr. 42); Perplex

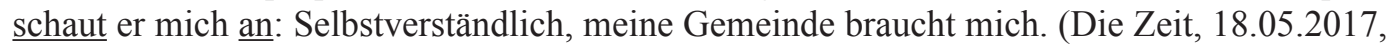
Nr. 18); Doch dann platzt die Internetblase, das Unternehmen geht pleite, und Thelen soll den Kredit abstottern. (Die Zeit, 27.12.2017, Nr. 53); Das Start-up ging nach nur 24 Monaten im Jahr 2000 spektakulär pleite. (Die Zeit, 13.12.2017 (online))

Von den derivatologischen Gründen, die gegen die Klassifikation der betreffenden Lexeme zu den Kopulapartikeln sprechen, sind mehrere zu nennen. Erstens: das zur Bildung von Adjektiven dienende und äußerst produktive Suffix -ig (vgl. Fleischer und Barz 1995: 39; Weinrich 1993: 993), das sich auch in Lexemen $a b$ spenstig, anheischig, vorstellig und teilhaftig findet und in Analogie zu den sonstigen Adjektiven die Deklination dieser Derivate ermöglicht (s. 2, 3, 9, 11) ${ }^{15}$. Zweitens: bei der Konversion eines Adjektivs in ein Nomen bewahren solche deadjektivischen und departizipialen Konversionsprodukte (von ein paar Ausnahmen abgesehen) die sexusabhängige Genusvariation und die adjektivische Flexion, wobei sie in maskulinen bzw. femininen Formen der Regel nach als Personenbezeichnungen fungieren, z.B. der/die Alte; der/die Liebste; der/die Angestellte; der/die Lesende u.a., während bei Nicht-Personenbezeichnungen in der Regel die neutrale Form

${ }^{14}$ Man vergleiche z.B. das Adjektiv fleißig in folgenden syntaktischen Funktionen: Der Student ist fleißig. (prädikativ) / Der fleißige Student. (attributiv) / Der Student lernt fleißig. (adverbial).

${ }^{15}$ Das einzige Lexem dieses Wortbildungsmusters, das auch weiterhin als Kopulapartikel zu betrachten sei, ist ausfindig, da es ausschließlich als Bestandteil der Wendung jemanden / etwas ausfindig machen auftaucht (s. 4.2. (35)). 
verwendet wird, z.B. das Alte, das Beste u.a. (Fleischer und Barz 1995: 215). Die durchgeführte Analyse hat gezeigt, dass auch folgende Lexeme in gleicher Art und Weise substantiviert und dekliniert werden können: gewillt, meschugge, plemplem, teilhaft(ig), untertan und vorstellig. Vgl.:

(22) Die Prämien werden hierbei nicht nur Gewillten aus relativ sicheren Länder angeboten, sondern vor allem bei denjenigen beworben, die aus Kriegsgebiete wie Syrien und dem Irak stammen”, ... (www.dielinke-nrw.de; 10.7.2018); „Wir behalten uns weiter das souveräne Recht vor, militärische Gewalt gegen Irak anzuwenden - allein oder in einer Koalition der Gewillten. (www.manager-magazin.de; 27.1.2003); Jeder ist zugleich ein Teufelskerl und Meschuggener oder, in der Sprache des Volkes, ein Mamser, und ,jeder Mamser ist ein König”. (Die Zeit, 08.11.1968, Nr. 45); Was das mit „frei” zu tun haben soll, ist wohl nur den völlig Plemplemen bekannt. (www.politnetz.ch; 10.10.2016); Wahrscheinlich ist der Anteil der Plemplemen ungefähr so hoch wie in jeder Bevölkerungsgruppe, aber es gibt hier inzwischen recht viele ... (www.comicforum.de; 3.11.2012.); Daraus, daß eine Meinung offenbar immer das Gegenteil einer anderen Meinung ausdrückt, schließe ich, daß eine Meinung nie das Ganze ausdrückt, daß sie also etwas Teilhaftes ist. (Die Zeit, 14.12.1990, Nr. 51); Immer neue Koseworte finden sie füreinander, und was für welche: sie, die Teilhaftige, Maestra, Gelieferte, Kapitulierende, er, der Ausgelieferte, der Belletrist, der Übermütige, Erschienener, von ihr Lebender, ihr ganz und gar Gehörender, ... (www.sueddeutsche.de; 10.9.2012); Zoll: Wie eine Behörde die Untertanen schikaniert (www.spiegel.de; 27.5.2015); Auf Bagdads Straßen fühlen sich Amerikas GIs wie lebende Zielscheiben, während die Untertanen des geflüchteten Diktators Saddam Hussein immer lauter Arroganz und Inkompetenz der Eroberer beklagen. (www.spiegel.de; 21.7.2003); Die Strategie des Finanzamtes ist, Veranlagungen vermehrt online abzuwickeln, weshalb das Expertenteam mit den Vorstelligen auch 61 Online-Anträge ausgefühlt hat. (www.rundschau.at; 13.3.2018); Die Vorstelligen von Lopavend waren mitte 20 und hauptberuflich Studenten aus Berlin, deren Aufgabe es war, die Anwohner der Karl-LehrStrasse auf der Dellviertelseite mit einer, zugegeben charmanten Inszenierung, zu beruhigen. (www.ruhrbarone.de; 24.7.2015)

Drittens stellen einige der analysierten Lexeme etymologisch betrachtet Ableitungen mit dem Adjektiv als Basis dar, wie z.B. abhold (mittelhochdeutsch abholt $=$ feindlich gesinnt, aus $a b$ und hold (Adj.): mittelhochdeutsch holt, althochdeutsch hold = günstig, gnädig; treu, vgl. Duden), was die Möglichkeit der Deklination erklären könnte.

3.3. Als Grenzfälle zwischen Adverbien und Adjektiven sind barfuß (2) und k.o. (3) zu betrachten. Sie kommen meist prädikativ vor (z.B. Er war völlig k.o.; Die Kinder waren barfuß.), können aber auch von Vollverben regiert werden, alleine im Vorfeld des Konstativsatzes stehen und als Antwort auf eine $w$-Frage dienen, wobei sie erwartungsgemäß die Position Det_Nom ausschließen sollten. Daher erfüllen sie alle für die Wortart Adverb aufgestellten Kriterien (vgl. Engel 2004: 411), wovon folgende Belege zeugen:

(23) Im Prinzip sind Barfußschuhe etwas, das gar nicht geht - man kann nicht zugleich barfuß gehen und Schuhe tragen. (Die Zeit, 06.01.2018, Nr. 02); Wenn er mit seinen Leuten im Kongo kämpfte, mussten die Kubaner die Schuhe ausziehen und barfuß kämpfen wie die einheimischen Baluba. (Zeit Magazin, 22.12.2017, Nr. 53); Wer Zehen hat, zu fühlen, möchte sich nach diesem Satz dafür verwünschen, noch nie barfuß in eine Biene getreten zu sein. (Die Zeit, 16.11.2017, Nr. 47); Er geht barfuß, sogar im Winter. (Die Zeit, 05.11.2017, Nr. 45) 
(24) Als er endgültig $k$. o. am Boden lag, haben sie mich ausgepfiffen. (Die Zeit, 08.07.2017, Nr. 28); Und zuvor hatte Rocky im vierten Teil schon Ivan Drago und den Ostblock k. o. gehauen, im Kampf des Jahrhunderts. (Die Zeit, 05.09.2013, Nr. 37); »Wenn ich dich wirklich hätte schlagen wollen, wärst du doch $k$. o. gegangen!« (Zeit Magazin, 22.12.2011, Nr. 52); Irgendwo in Bernhards gepanzertem Schädel muss die Fantasie spuken, Claudia $k . o$. zu schlagen; ... (Zeit Magazin, 22.12.2011, Nr. 52) ${ }^{16}$

In letzter Zeit macht sich auch die Tendenz zur Adjektivierung dieser Lexeme bemerkbar. Sie kann durch folgende Belege illustriert werden, die zwar nur sporadisch (barfuß) oder nur textsortenspezifisch (k.o.) auftauchen, aber zusätzlich gegen die Zuweisung von barfuß und k.o. zu den Kopulapartikeln sprechen:

(25) Als der barfuße Bargeld bei „Silence Is Sexy” pflichtgemäß eine Zigarette anzündete - ..-, inhalierten manche im Saal förmlich mit. (www.zeit.de; 23.1.2017); Bei dem herbstlichen Wetter war es für eine barfuße Wanderung ein wenig frisch. (www.dorstenerzeitung.de; 15.9.2008); Das barfuße Auftreten ohne Strümpfe und Schuhe war ein Markenzeichen von Césaria, welches sie auch später auf den großen Bühnen der Welt beibehielt. (https://algarve-entdecker.com; 6.5.2015); Mehr über barfuße Fortbewegung erfahren Sie auf gesund.at (www.meinbezirk.at; 27.7.2016)

(26) Gegen technisch starke hat er gar keine Chance. Er ist einfach nur ein $K O$ Boxer! (www.sofacoach.de; 18.11.2010.); Mit diesem KO Sieg, der das Publikum von den Sitzen riss, konnte Danyo Ilunga die Partien nun 2:1 für sich entscheiden. (www.rheinische-anzeigenblaetter.de; 15.12.2017); Entdecken Sie den K.O. Schlag, den der legendäre Shaolinmönche Yi Long mit einem fantastischen rechten Haken gegen einen brasilianischen Muay Thai Kämpfer ausführte. (www.gentside.de; 5.1.2016)

\section{DAS INVENTAR UND DIE VALENZ DER KOPULAPARTIKELN IM GEGENWARTSDEUTSCH}

Mit der langen Liste der Kopulapartikeln im Ausgangskorpus verglichen, konnte im Kontrollkorpus nur bei verhältnismäßig geringer Anzahl von Lexemen die Zugehörigkeit zu dieser Wortart bestätigt werden. Sie lassen sich in zwei Gruppen aufteilen.

4.1. Die erste Gruppe bilden: angst (3), feind (4), leid (4), schade (4), schuld (2) und schnuppe (4). Allen gemeinsam ist es, dass sie denominale Konversionsprodukte sind (z.B. die Angst $\rightarrow$ angst; das Leid $\rightarrow$ leid u. a.) und in Verbindung mit Kopulaverben immer kleingeschrieben werden. Weinrich (1993: 992) betrachtet solche aus Substantiven entstandenen Wörter als Konversionen Substantiv zu Adjektiv. Auch im Duden-Wörterbuch werden sie, wenn sie in Verbindung mit Kopulaverben sein, werden und bleiben auftreten, als Adjektive klassifiziert, die nach der neuen

${ }^{16}$ Wie aus den Beispielen ersichtlich, trifft der adverbiale Gebrauch des polysemen Lexems k.o. vorwiegend auf die für den Sportjargon (Boxen) charakteristische Bedeutungsvariante 'nach einem Niederschlag kampfunfähig und besiegt' zu, während die zweite, umgangssprachliche Bedeutungsvariante 'körperlich völlig erschöpft, übermüdet' meist prädikativ realisiert wird. Da sich aber bei k.o. um ein Lemma mit zwie Sememen handelt (vgl. Duden), wird in diesem Beitrag von einer Wortart ausgegangen. 
Rechtschreibung kleingeschrieben werden (z.B. Mir ist/wurde angst. Aber: Ich habe Angst.). Vom dependentiellen Modell U. Engels ausgehend weisen solche sprachlichen Einheiten alle für die Wortart Kopulapartikeln relevanten Merkmale auf: sie werden von den Kopulaverben als Prädikativergänzung regiert und schließen aufgrund der substantivischen Herkunft den attributiven Gebrauch aus, vor allem deswegen, weil die morphologischen Vorbedingungen für die Adjektivdeklination fehlen. Daher sind solche Konversionsprodukte nur prädikativ vorzufinden und eindeutig zu den Kopulapartikeln zu rechnen.

Die einzige Ausnahme von dieser Regel bildet das Lexem pleite, das ebenso zu den Desubstantiva zählt, aber vermutlich aus phonologischen Gründen - nämlich wegen des Graphems $e$ im Wortauslaut, das die Flexion erleichtert - auch in flektierter Form vorzufinden ist (s. 16). Ob dies einmal auf die gleich auslautenden Desubstantiva schade und schnuppe zutreffen wird, bleibt weiter zu verfolgen. Darüber hinaus wird in der einschlägigen Fachliteratur auch das Lexem gram öfter zu den Desubstantiva gerechnet (s. Weinrich 1993: 992). Ein Blick auf die Etymologie sagt uns, dass hier eigentlich eine Konversion in umgekehrter Richtung vorliegt - aus dem Adjektiv gram ist nämlich das Nomen der Gram entstanden ${ }^{17}$, so dass sich der attributive Gebrauch dieses Lexems mit dessen adjektivischer Herkunft erklären lässt (s. 7). Aus allen genannten Gründen werden in der vorliegenden Arbeit sowohl pleite als auch gram aus der Klasse der Kopulapartikeln ausgeschlossen und den Adjektiven zugerechnet.

Noch ein Desubstantivum musste aus der Liste der Kopulapartikeln entfernt werden, nämlich wett (3), das auf das Nomen Wette zurückzuführen ist (vgl. DWDS: wett). Die Etymologie zeigt, dass sich wett früher mit Kopulaverben sein und werden verbunden hat und nur pädikativ verwendbar war. Aus synchroner Sicht aber ist die Wendung [mit jemandem] wett sein (in der Bedeutung 'quitt') aus dem Gebrauch gekommen, so dass wett nur als Erstglied des zusammengesetzten Verbs wettmachen erscheint und in den meisten Wörterbüchern überhaupt nicht als eigenständiges Lexem angeführt wird: „Dass wett, das Engel ausschließlich in Verbindung mit dem Verb machen als Kopulapartikel beschreibt, in sechs der neun untersuchten Wörterbüchern nicht erscheint, dürfte niemanden überraschen, wenn man bedenkt, dass in Duden. Die deutsche Rechtschreibung (2006) nur die zusammengeschriebene Form wettmachen als trennbares Verb angegeben wird, die auch in allen untersuchten Wörterbüchern angeführt wird. Somit dürfte dieses Wort ohne weiteres nicht nur als Adjektiv, sondern auch als Kopulapartikel ausgeschlossen werden.” (Castell 2016: 183).

${ }^{17}$ Gram m. 'Erbitterung, tiefe Betrübnis, nagender Kummer', älter 'Unmut, Zorn' (Anfang 15. Jh.), 'Feindschaft' ( Luther), Substantivierung des prädikativ gebrauchten Adjektivs (vgl. DWDS: Gram). 
Im Folgenden werden nur diejenigen Desubstantiva dargestellt, die die Kriterien der Wortart Kopulapartikeln erfüllen, also: angst (3), feind (4), leid (4), schade (4), schuld (2) und schnuppe (4). In Rücksicht wird auch deren Valenz genommen, da Kopulapartikeln, genauso wie Adjektive und Adverbien, phrasenbildend sind und sowohl Ergänzungen als auch Angaben regieren können.

Die Kopulapartikel angst unterscheidet sich von dem homonymen Nomen die Angst dadurch, dass sie immer kleingeschrieben und mit Kopulaverben sein oder werden kombiniert wird (jemandem ist, wird [es] angst), während sich das Nomen die Angst mit Vollverben (z.B.jemand hat, bekommt Angst; etwas macht jemandem Angst, Angst erregen usw.) oder Präpositionen (z.B. in Angst um jemanden sein; aus Angst etw. verschweigen) verbindet und dabei immer großgeschrieben wird. Die Kopulapartikel angst bildet Phrasen und fordert obligatorisch eine Dativergänzung, fakultativ eine Graduativergänzung. Vgl:

(27) «Mir ist nicht angst», verkündete Kapitän Philipp Lahm, für den der Tiefschlag gegen Dortmund in der Bundesliga «nullkommanull» Rolle spielt. (Die Zeit, 16.04.2012 (online)); Mir wird ganz angst, wenn ich daran denke, wie das zu bewältigen sein soll, wenn ich wieder meinem üblichen Zehnstundentag nachgehe. (Die Zeit, 16.09.2004, Nr. 39); „Zwischen den Möglichkeiten und deren Umsetzung klafft eine Distanz, dass einem angst werden kann", sagt Schubert. (Der Tagesspiegel, 20.12.2004)

Die Kopulapartikel feind, mit dem homonymen Nomen der Feind begrifflich eng verbunden, gilt heute als gehoben und veraltend, daher wurde die Wendung $j-m /$ einer Sache feind sein/bleiben (mit der Bedeutung 'jemandem, einer Sache feindlich, ablehnend gegenüberstehen' bzw. 'jemandem, einer Sache feindlich gesinnt bleiben', vgl. Duden) im Kontrollkorpus kaum belegt. In älteren, vor allem religiösen Texten (z.B. in der Bibel) lässt sich der Ausdruck finden, sogar in der Komparativform feinder $^{18}$, die heute gleichfalls verschwunden ist. Aus den Beispielen, die mangels Pressebelege den Wörterbüchern und Fachliteratur entstammen, wird deutlich, dass feind eine obligatorische Dativergänzung regiert. Vgl:

(28) Die beiden waren sich schon immer feind (Duden); Er war mir seit langem feind. (Engel 2004: 421); Sie war dem Nachbarn schon immer feind. (Đurović 2017: 99); Von da an blieb er dem Alkohol feind. (Castell 2016: 179)

Die Kopulapartikel leid, gebildet aus dem gleichlautenden Nomen das Leid, kommt in den Wendungen jemanden, etwas / (geh.:) jemandes, einer Sache leid sein / werden in der Bedeutung 'jemandes, einer Sache überdrüssig sein / werden' vor (vgl. Duden) und regiert dann eine obligatorische Akkusativ- bzw. (geh.) Genitivergänzung. Darüber hinaus taucht sie als erste Konstituente des zusammengesetzten Verbs leidtun auf(z.B. Das tut mir leid.) und gleichbedeutend, aber veraltend, auch

${ }^{18}$ Vgl.: Dazu hatte Joseph einmal einen Traum und sagte seinen Brüdern davon; da wurden sie ihm noch feinder. (Die Bibel: Erstes Buch Mose, Genesis 30); Da sprachen seine Brüder zu ihm: Solltest du unser König werden und über uns herrschen? und sie wurden ihm noch feinder um seines Traumes und seiner Rede willen. (Die Bibel: Erstes Buch Mose). 
mit dem Verb sein (z.B. Meine zornige Äußerung ist mir leid., vgl. Duden), während die Variante mit einer obligatorischen Dativergänzung und einer fakultativen Präpositivergänzung (um+Akk) in der Bibel vorzufinden ist (z.B. Es ist mir leid um dich, mein Bruder Jonathan. (Bibel, AT), vgl. Đurović et al. 2017: 100). Z.B.:

(29) Vielleicht sind sie die ewigen Debatten leid. (Die Zeit, 24.10.2017, Nr. 43); Die Deutschen seien es leid, sich sagen zu lassen, dass sie verantwortlich seien für die Aufnahme von Flüchtlingen. (Die Zeit, 25.09.2017 (online)); Es könnte zur Routine werden, ich könnte es leid werden. (www.neuepresse.de; 10.8.2013); Ruder sprach von einer Geschichte nach dem Muster „,wir hauen drauf, die hauen drauf” und stellte fest, dass er dieser Sache leid sei. (www.badische-zeitung.de; 29.04.2015.)

Die Kopulapartikel schade ist aus dem Nomen der Schaden (die Nebenform der Schade wird heutzutage als veraltet empfunden) durch Konversion entstanden und kommt in folgenden Wendungen vor: schade sein; etw. schade finden; schade um j.n / etw. sein; zu schade für/zu etw. sein und jmd. sich [Dativ] zu schade für/zu etw sein. Daraus lässt sich schließen, dass die Kopulapartikel sowohl als Subjektsprädikativ (regiert von dem Verb sein) als auch als Objektsprädikativ (regiert von dem Verb finden) erscheint ${ }^{19}$ und selber entweder eine fakultative Präpositivergänzung (um+Akk) oder eine fakultative Graduativ- bzw. Normergänzung fordert, wobei die letztgenannte üblicherweise mit einer für/zu-Phrase ergänzt wird. Vergleiche:

(30) Es wäre nur schade, wenn ihnen niemand dazu die Chance gibt. (Die Zeit, 04.10.2017 (online)); Ich finde es schade, dass die lokale Fußballkultur bei einer WM kaum eine Rolle spielt. (Die Zeit, 04.01.2018 (online)); Mit dem gleichen Boykottargument müsste man allerdings auch - und um die wäre es ja doch irgendwie schade - auf alle Platten von David Bowie verzichten. (Die Zeit, 28.12.2017 (online)); Die Zeit war zu schade dafür. (Die Zeit, 27.11.2017, Nr. 48); Das wäre natürlich sehr schade und auch sehr dumm, weil sie dann sehr viele Wähler verlieren würden. (Die Zeit, 31.10.2017 (online)); Die Kanzlerin darf sich nicht zu schade sein, den Abgeordneten Rede und Antwort zu stehen." (Die Zeit, 30.10.2017 (online))

Die Kopulapartikel schuld ${ }^{20}$ darf nicht mit dem homonymen Nomen die Schuld verwechselt werden, vgl: [an etwas] schuld sein vs. [an etwas] die Schuld haben (Duden). Die Partikel schuld fordert eine fakultative Präpositivergänzung (an+Dat). Vergleiche:

(31) Die Studenten seien heute viel schlechter als früher, kaum jemand sei intrinsisch motiviert, und Bologna und die Ökonomisierung, ja, die seien ohnehin an allem schuld. (Die Zeit, 06.01.2018, Nr. 02); Sollte Trump nun aber den Deal aufkündigen, würde er dem Regime das perfekte Argument in die Hand geben: „Der Westen ist an allem schuld.” (Die Zeit, 04.01.2018 (online)); Dass vielleicht sogar Thielemanns Mutter, eine Immobilienmaklerin, sagen würde: Du bist doch selbst schuld! (Zeit Magazin, 03.01.2018, Nr. 02)

Die Kopulapartikel schnuppe ist aus dem Nomen die Schnuppe (norddeutsch, mitteldeutsch: verkohlter Docht (einer Kerze o. Ä.)) durch Konversion entstanden.

${ }^{19} \mathrm{Zu}$ den Termini Subjekts- und Objektsprädikativ s. Helbig und Buscha (1998: 539-543).

${ }^{20}$ Interessanterweise taucht schuld in nur 2 Grammatiken (aus den Jahren 2004 und 2017) als Kopulapartikel auf, obwohl es eindeutig die Merkmale dieser Wortart aufweist. 
So lässt sich die umgangssprachliche Wendung [jemandem] schnuppe sein (= [jemandem] einerlei, egal, gleichgültig sein) als '[für jemanden] wertlos wie eine Schnuppe sein' paraphrasieren (Duden) ${ }^{21}$. Aus den Beispielen geht hervor, dass die Kopulapartikel schnuppe fakultativ eine Dativ- und eine Graduativergänzung an sich binden kann. Vgl.:

(32) Den meisten Befragten, fast 40 Prozent, ist es dagegen völlig schnuppe, in welchem Outfit ihr Arzt sie behandelt, entscheidend sei schließlich die Kompetenz. (Die Zeit, 19.04.2016 (online); Aber den kleinen Gästen war das ziemlich schnuppe. (Der Tagesspiegel, 28.09.2004); Es ist mir völlig schnuppe, was die Motivation dahinter ist. (Berliner Zeitung, 17.12.2005); Wie sie aussehen ist ihnen egal, sonst trügen sie nicht so komische Hosen, wie es ihnen nach dem Spiel geht, ist auch schnuppe, sonst liefen sie nicht andauernd gegeneinander. (Der Tagesspiegel, 14.06.2003)

4.2. In die zweite Gruppe wurden die restlichen Kopulapartikeln eingeordnet, also diejenigen, die keine Desubstantiva sind. Von der Wortbildung her lassen sie sich überhaupt nicht, semantisch nur teilweise auf einen gemeinsamen Nenner bringen. Gemeinsam ist allen nur, dass sie ausschließlich als Bestandteile fester Wendungen erscheinen. Zu dieser Gruppe gehören: los (4), quitt (4), ausfindig (4), eingedenk (4), egal (4) und einerlei (4), die im Folgenden einzeln dargestellt werden.

Die polyseme Kopulapartikel los wird einerseits in der Bedeutungsvariante 'nicht mehr fest [gehalten], gelöst, abgetrennt' realisiert (z.B.: Der Knopf ist los.) und andererseits als Bestandteil fester Wendungen j-n/etw. los sein; etwas ist los (mit j-m) und es ist viel / wenig / nichts los, in denen die semantische Motivation zwar verblasst, aber immer noch erkennbar ist, z.B.: j-n/etw. los sein bedeutet eigentlich 'von jemandem, einer Sache befreit sein', während die Ausdrücke [et] was ist los (mit j-m) und es ist viel / wenig / nichts los aus der Vorstellung entstanden sind, etwas habe sich gelöst, sodass es nicht mehr funktioniert (vgl. Duden: los). Das erste Semem (Der Knopf ist los.) steht in enger semantischer Beziehung zum Adjektiv lose (z.B. Der Knopf ist lose.), das ursprünglich als Nebenform von los betrachtet wurde (Fleischer und Barz 1995: 339). Gegenwärtig sind diese zwei gleichbedeutenden Formen morphologisch und syntaktisch strikt voneinander zu trennen, weil los unveränderlich und nur auf prädikativen Gebrauch beschränkt ist, daher als Kopulapartikel gilt, während lose auch attributive Funktion ausüben kann (ein loser Knopf; lose aufgestecktes Haar), steigerbar (lose, loser, am losesten) sowie graduierbar ist (zu/sehr/ziemlich lose) und somit zu den Adjektiven zählt. Des Weiteren muss man sich die Tatsache vor Augen halten, dass los nur in Verbindung mit dem Kopulaverb sein getrennt geschrieben wird (los sein) und als

${ }^{21}$ Für die Entstehung des homonymen Nomens die Schnuppe findet sich folgende Erklärung: „Früher musste man den abgebrannten Kerzendocht mit einer besonderen Lichtputzschere beschneiden, einen Vorgang, den man auch „schnupfen” oder „schnuppen” nannte. Den Dochtabfall bezeichnete man daher als „Schnuppe”, wobei die völlige Wertlosigkeit dieses Überbleibsels zur Redensart führte, die seit ungefähr 1850 in Berlin belegt ist. Die Sternschnuppe stellte man sich als Reinigungsabfall von Sternen vor." (www.redensarten-index.de). 
Kopulapartikel zu interpretieren ist, während es in allen anderen Realisierungen als homonyme Verbpartike ${ }^{22}$ fungiert, die polysem ist und Folgendes bezeichnen kann (vgl. DWDS: los-): 1) den Beginn der im Verb ausgedrückten Handlung (losbellen, loseilen, losfeuern, losfluchen, losgrölen, loslaufen usw.); 2) die Richtung auf ein Ziel hin (z.B. losfahren, losgehen, loslaufen, losmarschieren, losrennen, losziehen usw.); 3) eine Trennung (z.B. losbinden, losdrehen, loshaken, loskoppeln, loslösen, losschnallen, losschrauben usw.) und 4) die Befreiung aus einer bedrängten Lage (z.B. losbeten, losbitten, loskaufen, loskommen, lossprechen, loswerden usw.). Darüber hinaus kann das jeweilige Verb des Öfteren in der Oberflächenstruktur getilgt werden, da es leicht zu erschließen ist, z.B.: er ist mit dem Wagen los (losgefahren); ich will schon los (losgehen); ich habe die Schraube, das Brett schon los (losgedreht) usw. (vgl. Duden). Am Ende sei noch die homonyme Initiativpartikel los erwähnt, die in der Bedeutung 'schnell!; ab!' (als Aufforderung) erscheint (z.B.: los, beeil dich!, vgl. Duden) ${ }^{23}$.

Im Folgenden werden Beispielsätze für die Kopulapartikel los angegeben. Sie ist valenzfähig und fordert je nach der zu realisierenden Bedeutungsvariante entweder eine obligatorische Akkusativergänzung ( $j$-n/etw. los sein) oder eine fakultative Präpositivergänzung mit+Dat (etwas ist los (mit j-m)), ggf. auch von+Dat (Der Hund ist (von der Kette) los.), während die situative Bestimmung als Situativangabe fungiert und daher immer fakultativ ist. Andererseits taucht das Vorfeld-es in den Wendungen es ist viel / wenig / nichts los als Platzhalter und steht für das eigentliche Subjekt viel / wenig / nichts, das jederzeit die erste Position im Satz einnehmen und den Wegfall des Platzhalters verursachen kann (Nichts/Viel/Wenig ist los.). Zur Illustration vgl.:

(33) Wer einmal das Brandmal ,geistig behindert” trägt, wird es nicht wieder los. (Die Zeit, 04.01.2018, Nr. 01); Nicht unbedingt, aber die Schatten von 1942 wird die Ufa nie wieder los. (Die Zeit, 30.12.2017, Nr. 01); „Was ist mit dir los?” (Die Zeit, 04.01.2018, Nr. 01); „Wobei meine Mama ja eigentlich gar nicht wusste, was bei uns zu Hause los war, weil sie ja den ganzen Tag bei Emil war." (Die Zeit, 13.03.2018, Nr. 19); Vielleicht weil in meinem Kopf so viel los ist. (Die Zeit, 08.01.2018, Nr. 02); Einerseits gab es die Straße, da war immer was los. (Der Tagesspiegel, 05.06.2005); Es war einfach nichts los. (Der Tagesspiegel, 01.06.2005)

${ }^{22}$ Fleischer und Barz (1995: 339) betrachteten los- früher als Präfix und sprachen von den Präfixderivaten (losfahren, losfliegen usw.). Dagegen wird in der neuesten Auflage (vgl. Fleischer und Barz 2012: 419, 421) los- als (adverbiale) Verbpartikel interpretiert, in Anlehnung an E. Donalis (2005: 29), die Partikelverben aufgrund der syntaktischen Mobilität deren Bestandteile als eigenständige Wortbildungsform betrachtet. Die Autorin geht nämlich von der Annahme aus, dass Derivation und Komposition untrennbare Wörter bilden. Motsch (1999: 45-48) unterscheidet zwar untrennbare Präfixe von trennbaren Verbpartikeln, betrachtet aber beide als Affixe (vgl. los, S.107). Kühnhold und Wellmann (1973: 227; 288) erfassen los- als Präfixoid.

${ }^{23}$ Initiativpartikeln werden in der Fachliteratur als ein Untertyp der Satzäquivalente betrachtet, die keine Äußerungen anderer Personen voraussetzen, sondern vielmehr verwendet werden, um ein bestimmtes Verhalten des Partners herbeizuführen: „Durch los! kann man eine andere Person oder andere Personen zum Handeln veranlassen.” (Đurović et al. 2017: 209f.) 
Die Kopulapartikel quitt ist lateinischer Herkunft (mittelhochdeutsch quît $<$ altfranzösisch quite < lateinisch quietus = ruhig; untätig; frei (von Störungen), vgl. Duden) und tritt im Gegenwartsdeutsch als Prädikativergänzung der Kopulaverben sein und werden auf oder als Teil der Wendung mit jemandem quitt sein /werden in der Bedeutung 'frei von allen Verbindlichkeiten, Schulden'. Als veraltend gilt heute der Genitiv in der Wendung jmds./einer Sache quitt sein/werden mit der Bedeutung 'jmdn., eine Sache los sein, werden' (z.B. seines Amtes quitt sein, werden, vgl. DWDS: quitt), während die gleichbedeutende Wendung im Akkusativ $j$ - $n$ / etw. quitt werden zwar selten, aber immer noch in Gebrauch ist. Je nach der zu realisierenden Bedeutungsvariante regiert quitt entweder eine fakultative Präpositivergänzung (mit+Dat) oder (seltener) eine obligatorische Akkusativergänzung. Vgl.:

(34) Ich empfinde das als brüderliche Geste, wir sind quitt.» (Die Zeit, 14.02.2013 (online)); Wenn wir Kabinettsdiplomatie im Stil des neunzehnten Jahrhunderts treiben könnten, würde man Afghanistan entweder als neutralen Pufferstaat wiederherstellen oder anderswo mit den Russen quitt werden. (Die Zeit, 08.02.1980, Nr. 07); ,Wenn wir beide zwei Menschenleben retten, sind wir quitt mit Hitler, und jedes zusätzliche Leben ist ein Reingewinn." (Die Zeit, 05.02.2004, Nr. 07); Hat er seine Frau nach Wien geschickt, um sie quitt zu werden? (Die Welt, 06.12.2003)

Die Kopulapartikel ausfindig kommt im Gegenwartsdeutsch ausschließlich als Bestandteil der Wendung jemanden, etwas ausfindig machen mit der Bedeutung 'jemanden, etwas [lange, mühsam] suchen und schließlich finden' vor und ist nicht phrasenbildend. Der attributive Gebrauch, der durch das Adjektivsuffix -ig zu erwarten wäre (s. 3.2.), ist nur in älteren Texten (aus dem 17., 18. oder 19. Jh.) vorzufinden, während sporadische Beispiele im Gegenwartsdeutsch eher von einem fehlerhaften Gebrauch zeugen ${ }^{24}$. Ob sich die Tendenz zur Adjektivierung dieser Kopulapartikel weiter entwickeln wird, bleibt zu verfolgen. Vgl.:

(35) "Manche Hausbesitzer", sagt er, "waren überhaupt nicht mehr ausfindig zu machen." (Die Zeit, 18.11.2017 (online)); Nur so könne man ökonomische Reserven ausfindig machen, meint der Senat. (Der Tagesspiegel, 29.04.2005); Beamte konnten die drei Tatverdächtigen schließlich im Imbiss ausfindig machen. (Berliner Zeitung, 13.09.2005)

Die Kopulapartikel eingedenk erscheint als Bestandteil der Wendung einer Sache eingedenk sein/bleiben mit der Bedeutung 'sich an etwas erinnern und es beherzigen, sich etwas vor Augen halten', die mit dem stilistischen Merkmal gehoben versehen und daher in den hier untersuchten Textsorten nicht sehr häufig in Gebrauch ist. Die homonyme Präposition eingedenk (Genitiv) mit der Bedeutung 'unter Berücksichtigung einer Sache; wegen' gilt gleichfalls als gehoben (vgl.

${ }^{24}$ Z.B.: Für jene, die Eigenarten und Gewohnheiten der italienischen Märkte erkunden, aber auch für ausfindige Schnäppchenjäger, die gerne ein gutes Geschäft abschließen wollen, sind diese Ereignisse eine unentbehrliche Erfahrung. (www.enit.de/reisethemen/maerkte); Die ausfindige Person gehört wegen schwerer Nötigung angezeigt und verhaftet! (www.kleinezeitung.at/; 10.04.2016; Kommentar); Die ausfindige Firma könnte am Samstag mit dem Bau beginnen, sie benötigen etwa 2-3 Tage dafür. (www.facebook.com/animalsoulsavior/). 
Duden: eingedenk), ist aber in der Pressesprache des Öfteren belegt. Hier ein paar Beispiele für die Kopulapartikel eingedenk, die obligatorisch eine Genitivergänzung fordert, sporadisch aber auch eine Akkusativergänzung regieren kann:

(36) Ist man der Würde eingedenk, mit der Dieter Henrich, nicht zuletzt seinen Eltern dankend (...), den Hegel-Preis entgegennahm, dann fällt es schwer, skeptisch von der Philosophie zu sprechen. (Die Zeit, 22.12.2003, Nr. 01); Lasst uns Weihnachten zum Anlass nehmen, wieder der alten Botschaft eingedenk zu werden: (Die Zeit, 17.01.2002, Nr. 04); Das aber bedeutet, nach einer Medizin zu fragen, die der Einheit von Heilen und Lindern, von aktiver Intervention und behutsamen Beistand, in jeder ihrer Handlungen eingedenk wäre. (Berliner Zeitung, 04.05.2001); Streitenden ging er, das Schicksal seines Ahnen eingedenk, aus dem Weg, wohl aber sah er sich im Fernsehen gern Übertragungen von Fußballspielen an. (Berliner Zeitung, 11.08.2001)

Die zwei gleichbedeutenden Kopulapartikeln egal und einerlei sind nur in der Bedeutung 'gleichgültig' (z.B. Das ist mir egal / einerlei.) als solche zu definieren und strikt von den homonymen und gleichbedeutenden Adjektiven egal und einerlei mit der Bedeutung 'gleichartig' zu trennen. Die Adjektive unterscheiden sich untereinander nur dadurch, dass egal in attributivem Gebrauch flektiert, einerlei dagegen stets unflektiert erscheint ${ }^{25}$. Darüber hinaus soll auf das homonyme, nur landschaftlich, besonders ostmitteldeutsch, salopp gebrauchte Adverb egal hingewiesen werden, das die Bedeutung 'immer, fortwährend' hat (z.B. er hat egal Hunger; ist egal müde; dort regnete es egal; du musst nicht egal meckern!, vgl. DWDS: egal). Hier ein paar Beispiele für die Kopulapartikeln egal und einerlei, die eine fakultative Dativergänzung regieren können, welche durch die gleichbedeutende für-Phrase ersetzbar ist. Dazu kommt oft eine fakultative Graduativergänzung. Vergleiche:

(37) "Es ist völlig egal, woher du kommst", erklärt Sohm. (Zeit Magazin, 04.01.2018, Nr. 01); Als cool galt das seltsame Hobby bei den Jungs seiner Umgebung nicht, aber das war ihm egal. (Die Zeit, 08.01.2018, Nr. 02); Die Typen aus der Werkstatt hatten der Polizei gesagt, dass sie den Besitzer nicht kennen, und dass ihnen egal sei, was mit der alten Karre passiert. (Der Tagesspiegel, 06.06.2005); Dem Publikum ist es nämlich völlig egal, ob ein Schauspieler mehr "wert" ist, als ein anderer. (Berliner Zeitung, 29.12.2005)

(38) Niemand würde behaupten, es sei ihm einerlei, ob ihm der Geldbeutel mit oder ohne Gewalt weggenommen wird. (Die Zeit, 05.12.2017 (online)); Den meisten Russen ist es

${ }^{25}$ Als Adjektive sind egal und einerlei in folgenden Belegen zu interpretieren: Nach seinen sehr egalen jüngsten Alben, das letzte erschien vor sieben Jahren, konzentrierte sich Adams auf seine Karriere als Fotograf und Magazin-Herausgeber, ... (www.spiegel.de; 20.10.2015); Wenn man sich nicht von der egalen Musik ablenken lässt, besitzt diese Fernsehgarten-Black-Metal-Band absolutes Siegerpotenzial. (www.spiegel.de; 11.5.2018); Wäre das nicht so, würden sich die Patriarchen mit dem Herrschen viel schwerer tun und wir dem Licht am fernen Horizont - einer egalen Gesellschaft - näher sein. (https://derstandard.at; 16.12.2008); „Ist es aber nur von einerlei Spielzeug, Filmen und Büchern umgeben, dann kennt es nur die eine Hälfte der Welt und bleibt in seinen Möglichkeiten eingeschränkt. (Zeit Magazin, 07.12.2017 (online)); Wenn sie meinen, man müsse einen ungehörten Buchstaben wegen unterschiedlicher Bedeutung einiger Wörter, die einerlei Klang haben, schreiben, so kann man ihnen, ..., hundert Beispiele darlegen, da Wörter von sehr verschiedener Bedeutung von ihnen selbst mit einerlei Buchstaben geschrieben werden. (Die Zeit, 31.12.2004, Nr. 01) 
nach Umfragen einerlei, wer kommt. (Die Zeit, 29.04.2015, Nr. 18); Für den IS ist es einerlei, für uns sollte es das nicht sein. (Die Zeit, 17.12.2014, Nr. 52); Ob er Revolutionär oder Tyrann oder gleich beides wird, bleibt einerlei. (Die Zeit, 11.05.2005, Nr. 20)

\section{SCHLUSSFOLGERUNG}

Die durchgeführte Analyse hat gezeigt, dass die Zahl sämtlicher Kopulapartikeln im Gegenwartsdeutsch überraschend klein ist. Von insgesamt 33 Lexemen, die im Ausgangskorpus in wenigstens zwei Werken als Kopulapartikeln klassifiziert wurden, weisen 19 die Merkmale der Wortart Adjektiv auf, da sie deklinierbar und nicht selten auch komparierbar sind, daher nicht nur prädikativ, sondern auch attributiv und ggf. auch adverbial gebraucht werden können. Darüber hinaus weisen sie des Öfteren auch formale Übereinstimmungen mit den Adjektiven auf oder sie sind etymologisch als solche zu interpretieren. Zwei Lexeme sind als Grenzfälle zwischen Adjektiven und Adverbien zu betrachten und deren syntaktisches Verhalten bedarf weiterer Untersuchung. In Anbetracht dessen konnte für die deutsche Gegenwartssprache ein Inventar von insgesamt 12 Kopulapartikeln nachgewiesen werden, die zum einen in Homonymiebeziehungen zu den Nomen stehen, aus denen sie durch Konversion entstanden sind (angst, feind, leid, schade, schuld und schnuppe), zum anderen als Bestandteile fester Wendungen fungieren (los, quitt, ausfindig, eingedenk, egal und einerlei) und teilweise in Homonymiebeziehungen zu anderen Wortarten (los, egal und einerlei), teilweise in Synonymiebeziehungen zueinander (einerlei und egal; quitt und los) stehen. Allen Kopulapartikeln ist gemeinsam, dass sie ausschließlich prädikative Funktion im Satz ausüben und weder dekliniert noch kompariert werden können, also zu den Unflektierbaren gehören, was die entscheidenden Kriterien für deren Abgrenzung von Adjektiven und Adverbien sind. Mit den Adjektiven und Adverbien haben sie gemeinsam, dass sie phrasenbildend und valenzfähig sind. In einer Kopulapartikel-Phrase kommen reine Kasusergänzungen und Präpositivergänzung vor. Des Weiteren können manche von ihnen aufgrund deren Semantik mit Hilfe von lexikalischen Mitteln graduiert werden und eine Graduativergänzung regieren, wobei sie selber immer unflektiert bleiben. 


\section{KORPUS}

DWDS-Zeitungskorpora:

Berliner Zeitung (1994-2005)

Tagesspiegel (1996-2005)

Die Zeit (1946-2018)

Tschick - Herrndorf, Wolfgang: Tschick. Roman. 5. Auflage. Berlin: Rowohlt Taschenbuch Verlag, April 2012.

Meist zitierte Internetquellen:

https://derstandard.at

www.derstandard.de

www.deutschlandfunk.de

www.faz.net

www.focus.de

www.fr.de

www.n-tv.de

www.ruhrnachrichten.de

www.spiegel.de

www.sueddeutsche.de

www.taz.de

www.welt.de

www.zeit.de

\section{LITERATURVERZEICHNIS}

Castell 2016: A. Castell: Abhold, leid, gewillt, ... Sind das Adjektive? Zur Erstellung eines Korpus für ein syntaktisches Adjektivwörterbuch Deutsch-Spanisch. In: Revista de Filologia Alemana, Bd. 24, 2016: 177-191. <https://revistas.ucm.es/index.php/RFAL/article/viewFile/52823/48515> 16.05.2018.

Denkler et al. 2008: M. Denkler / S. Günthner / W. Imo / J. Macha / D. Meer / B. Stoltenburg / E. Topalović (Hrsg.): Frischwärts und unkaputtbar. Sprachverfall oder Sprachwandel im Deutschen. Münster: Aschendorff Verlag, 2008. 
Donalis 2005: E. Donalis: Die Wortbildung des Deutschen. Ein Überblick. 2., überarbeitete Auflage, Tübingen: Gunter Narr Verlag, 2005.

Duden: Duden-Online-Wörterbuch <www.duden.de>

Duden 2005: Duden: Die Grammatik. Unentbehrlich für richtiges Deutsch. 7., völlig neu erarbeitete und erweiterte Auflage. Band 4. Mannheim: Dudenverlag, 2005.

Đurović et al. 2017: A. Đurović / B. Ivanović / S. Srdić / U. Engel / M. Alanović: Deutsch-serbische kontrastive Grammatik. Teil IV: Partikeln. Hamburg: Dr. Kovač Verlag, 2017.

Engel und Mrazović 1986: U. Engel / P. Mrazović (Hrsg.): Kontrastive Grammatik Deutsch-Serbokroatisch. Mannheim: IDS, Novi Sad: Philosophische Fakultät, 1986.

Engel 1988: U. Engel: Deutsche Grammatik. Heidelberg: Julius Groos Verlag, 1988.

Engel 2004: U. Engel: Deutsche Grammatik. Neubearbeitung. München: Iudicium Verlag, 2004.

Fleischer und Barz 1995: W. Fleischer / I. Barz: Wortbildung der deutschen Gegenwartssprache. 2., durchgelesene und ergänzte Auflage. Tübingen: Max Niemeyer Verlag, 1995.

Fleischer und Barz 2012: W. Fleischer / I. Barz: Wortbildung der deutschen Gegenwartssprache. 4., völlig neu bearbeitete Auflage. Berlin/Boston: De Gruyter, 2012.

Helbig und Buscha 1998: G. Helbig / J. Buscha: Deutsche Grammatik. Ein Handbuch für den Ausländerunterricht. Leipzig: Langenscheidt Verlag Enzyklopädie, 1998.

Hentschel und Weydt 2003: E. Hentschel / H. Weydt: Handbuch der deutschen Grammatik. 3., völlig neu bearbeitete Auflage. Berlin: Walter de Gruyter, 2003.

Hrustić 2006: M. Hrustić: Kopulativni glagoli i kopulativna partikule u njemačkom i u bosanskom jeziku, u: Pismo IV/1, Sarajevo: Bosansko filološko društvo, 130-146.

Keller 2006: R. Keller: Ist die deutsche Sprache vom Verfall bedroht?, S. 193-205. $<$ http://www.phil-fak.uni-duesseldorf.de/rudi.keller/downloads/ Sprachverfall.pdf $>$ 27.02.2018.

Kühnhold und Wellmann 1973: I. Kühnhold / H. Wellmann: Deutsche Wortbildung. Typen und Tendenzen in der Gegenwartssprache. Das Verb. Düsseldorf: Pädagogischer Verlag Schwann, 1973. 
Motsch 1999: W. Motsch: Deutsche Wortbildung in Grundzügen. Berlin: Walter de Gruyter, 1999.

Petronijević 2011: Б. Петронијевић: Категоријална обележја партиципа I у немачком и српском језику, Српски језик, XVI, 21-39.

Römer 2006: C. Römer: Morphologie der deutschen Sprache. Tübingen: A. Francke Verlag, 2006.

Trost 2006: I. Trost: Das deutsche Adjektiv. Untersuchungen zur Semantik, Komparation, Wortbildung und Syntax. Hamburg: Helmut Buske Verlag, 2006.

Wahrig 2009: Wahrig: Richtiges Deutsch leicht gemacht. Band 5. Wissenmedia Verlag, 2009.

Weinrich 1993: H. Weinrich: Textgrammatik der deutschen Sprache. Mannheim / Leipzig / Wien / Zürich: Dudenverlag, 1993.

Zifonun et al. 1997: G. Zifonun / L. Hoffmann / B. Strecker: Grammatik der deutschen Sprache. Band 1. Berlin: Walter de Gruyter, 1997.

КОПУЛАТИВНЕ ПАРТИКУЛЕ У САВРЕМЕНОМ НЕМАЧКОМ ЈЕЗИКУ

Резиме

У раду се испитује статус језичких јединица које се у депенденцијалном моделу У. Енгела издвајају у посебну класу непроменљивих врста речи - копулативне партикуле. Њихова главна карактеристика и дистинктивно обележје у односу на друге класе речи јесте предикативна функција као једини могући вид реализације, што се у савременом (не само разговорном) немачком језику не потврђује увек, већ се запажа тенденција њихове употребе и у другим синтаксичким функцијама, пре свега атрибутској, што за резултат има прелазак датих лексема из класе непроменљивих у класу променљивих врста речи, најчешће придева. Истраживање спроведено на корпусу који чине текстови публицистичког функционалног стила потврдило је почетну хипотезу да је инвентар копулативних партикула услед описаних тенденција далеко мањи него што се то у стручној литератури обично наводи.

Кључне речи: копулативна партикула, придев, прилог, валентност, немачки језик.

Даница Б. Недељковић 